\title{
STRESS IN THE VICINITY OF A CRACK IN A THICK ELASTIC PLATE*
}

\author{
BY \\ M. LOWENGRUB \\ The Department of Mathematics, Duke University
}

1. Introduction. The determination of the distribution of stress in the vicinity of a crack plays a central part in recent theories of fracture [1], [2] and for that reason is of some technical importance. Much of this work is based upon an analysis of the stress near a circular or "penny-shaped" crack first discussed by Sneddon [3]. In that paper Sneddon reduced the problem of finding the stress near a penny-shaped crack in an infinite elastic solid to the determination of the solution of a pair of dual integral equations. Green [4], recognizing the relation of this problem to that of the electrified disc problem of electrostatics (see, for instance, p. 175 of [5]) suggested a different approach leading to the solution of an integral equation. A solution of this crack problem using the ideas of Green and employing oblate spheroidal coordinates has also been given by Payne [6]. Still another approach involving this latter coordinate system is due to Sack [7].

All of these discussions are confined to the case of an infinite solid and only one of them - that due to Sneddon-appears to lend itself to generalization to the case where the crack is situated in a thick plate. Even then the calculations can only be carried out when the crack is lying in the central plane of the plate with its surfaces initially parallel to those of the plate. In this paper we carry out this extension of Sneddon's method for the case in which the thickness of the plate is $\delta$ times the diameter of the circular crack.

As in Sneddon's original paper it is assumed that the equations of the classical (infinitesimal) theory of elasticity hold. The boundary conditions appropriate to two types of problem are formulated in Sec. 2. In Sec. 3 the problem is then reduced to the solution of a pair of dual integral equations which are solved in Sec. 4 by a procedure due to Lebedev and Uflyand [8]. Exact analytical expressions are derived for the solution only in the case of large values of $\delta$-the solution is correct to order $\delta^{-6}$ in $\delta$. The procedure to be followed in a numerical discussion of the solution is illustrated in Sec. 6 by the calculation of the shape of the deformed crack.

2. The boundary value problems. We shall determine the distribution of stress in the vicinity of a penny-shaped crack in an elastic plate of finite thickness but infinite radius. The crack will be taken to lie in the central plane of the plate with its surfaces parallel to those of the plate. We shall assume that the deformation occurs as the result of the application of prescribed forces or displacements to the plane boundaries of the plate, these forces being distributed symmetrically about an axis which passes through the center of the crack and is perpendicular to the plane of the crack. If we take the radius of the crack to be $a$, the crack therefore occupies the region $0 \leq r \leq a, z=0$, where $(r, \theta, z)$ are cylindrical coordinates referred to the center of the crack as origin

${ }^{*}$ Received September 13,1960 . The results contained in this paper were obtained in the course of an investigation conducted under contract No. AF 18(600) 1341 with the Air Force Office of Scientific Research of the Air Research and Development Command. 
and having the $z$-axis along the axis of symmetry of the problem. In this coordinate system we take the components of the displacement vector to be $(u, 0, w)$-the $\theta$-component being identically zero because of the symmetry-and the non-vanishing components of the stress tensor to be $\sigma_{r}, \sigma_{\theta}, \sigma_{z}, \tau_{r z}$. The thickness of the plate will be taken to be $2 d$.

In the analysis of the boundary value problems we make use of a technique first introduced by Sneddon [3] whereby a crack problem for the thick plate $-d \leq z \leq d$ is converted into a mixed boundary value problem for the elastic layer $0 \leq z \leq d$.

If we assume that the surfaces of the plate are given a uniform displacement $\epsilon$, then we have the boundary conditions

$$
\begin{array}{ccc}
\text { on } z=d: & w=\epsilon, & \tau_{r z}=0, \quad r \geq 0 . \\
\text { on } z=0: & \bar{\tau}_{r z}=0, & r \geq 0 ; \\
& \sigma_{z}=0, & 0 \leq r \leq a ; \\
& w=0, & r \geq a .
\end{array}
$$

The penultimate equation of this set is valid if there is no pressure applied to the surfaces of the crack, and the last one is a consequence of the prevailing symmetry. If we take the components of the displacement vector to be $(u, 0, w+\epsilon z / d)$ we see that $u$ and $w$ must be such as to lead to a displacement field satisfying the conditions

$$
\begin{aligned}
\text { on } z=0: \quad w & =0, \quad r \geq a ; \\
\tau_{r z} & =0, \quad r \geq 0 ; \\
\sigma_{z} & =-\frac{1-\nu}{(1+\nu)(1-2 v)}\left(\frac{\epsilon}{d}\right) E, \quad 0 \leq r \leq a . \\
\text { on } z=d: \quad w & =0, \quad r>0 ; \\
\tau_{r z} & =0, \quad r>0 .
\end{aligned}
$$

In Eq. (3), $\nu$ denotes Poisson's ratio and $E$ denotes Young's modulus.

On the other hand, if we assume that the free faces of the plate are pulled by a uniform tension $F$, we have the boundary conditions (2) again but the conditions (1) are replaced by:

$$
\text { on } z=d: \quad \sigma_{z}=-F, \quad \tau_{r z}=0, \quad r \geq 0 .
$$

In this case, if we take the components of the displacement vector to be

$$
\left\{u, 0, w-\frac{(1+\nu)(1-2 \nu)}{1-\nu}\left(\frac{F z}{E}\right)\right\},
$$

we must choose the functions $u, w$ to lead to a field satisfying the boundary conditions (3), (4), (7) and the conditions

$$
\begin{aligned}
\text { on } z=0: & \sigma_{z}=-F, & 0<r<a . \\
\text { on } z=d: & \sigma_{z}=0, & r>0 .
\end{aligned}
$$


3. The solution of the equilibrium equations. It is well-known ([9], p.452) that the expressions

$$
\begin{gathered}
u=-(1-2 \nu)^{-1} \frac{\partial^{2} \phi}{\partial r \partial z} \\
w=(1-2 \nu)^{-1}\left[2(1-\nu) \nabla^{2} \phi-\frac{\partial^{2} \phi}{\partial z^{2}}\right],
\end{gathered}
$$

satisfy the equations of elastic equilibrium and the compatibility relations, provided that $\phi$ satisfies the biharmonic equation

$$
\nabla^{4} \phi=0 .
$$

Corresponding to these components of displacement we have the expressions

$$
\begin{gathered}
\sigma_{z}=\frac{E}{(1+\nu)(1-2 \nu)}\left\{(2-\nu) \frac{\partial}{\partial z}\left(\nabla^{2} \phi\right)-\frac{\partial^{3} \phi}{\partial z^{3}}\right\}, \\
\tau_{r z}=\frac{E}{(1+\nu)(1-2 \nu)}\left\{(1-\nu) \frac{\partial}{\partial r}\left(\nabla^{2} \phi\right)-\frac{\partial^{3} \phi}{\partial r} \frac{\partial}{\partial z^{2}}\right\},
\end{gathered}
$$

for two of the stress components. The remaining two are given by similar expressions. Now the function

$$
\begin{aligned}
\phi=a^{3} \int_{0}^{\infty} \xi^{-2}\left\{\left[2 \nu A+\frac{(B+A \xi \delta) \xi \zeta \sinh \xi \delta}{2 \nu \sinh \xi \delta+\xi \delta \cosh \xi \delta}\right]\right. & \cosh \xi \zeta \\
& -(B+A \xi \zeta) \sinh \xi \zeta\} J_{0}(\xi \rho) d \xi,
\end{aligned}
$$

where $A$ and $B$ are arbitrary functions of $\xi$, and

$$
\rho=r / a, \quad \zeta=z / a, \quad \delta=d / a
$$

satisfies the biharmonic equation (13) and it is readily verified that it also leads to an expression for $\tau_{r z}$ which satisfies the boundary conditions (4) and (7).

4. The dual integral equations. We shall consider now the first of the two boundary value problems discussed in Sec. 2. The expression (16) satisfies the boundary conditions (4) and (7). It remains to determine the functions $A(\xi), B(\xi)$ so that the conditions (3), (5) and (6) are also satisfied.

If we substitute from Eq. (16) into Eq. (12) and make use of the condition (6), we find, after some manipulation, that, if the function (16) is such that the boundary condition (6) is satisfied then $A(\xi), B(\xi)$ must be related through the equation

$$
(\xi \delta+2 \nu \sinh \xi \delta \cosh \xi \delta) A(\xi)=\sinh ^{2} \xi \delta B(\xi) .
$$

We may therefore write

$$
A=-(1-\nu) a^{-1} \epsilon \psi(\xi), \quad B=-(1-\nu) \frac{\xi \delta+2 \nu \sinh \xi \delta \cosh \xi \delta}{\sinh ^{2} \xi \delta} a^{-1} \epsilon \psi(\xi)
$$

to obtain the expression

$$
\begin{aligned}
\phi=-(1-\nu) \epsilon a^{2} \int_{0}^{\infty} \xi^{-2} \psi(\xi) & {[\{2 \nu+\xi \zeta \operatorname{coth} \xi \delta\} \cosh \xi \zeta} \\
& \left.-\left\{\xi \delta \operatorname{cosech}^{2} \xi \delta+2 \nu \operatorname{coth} \xi \delta+\xi \zeta\right\} \sinh \xi \zeta\right] J_{0}(\xi \rho) d \xi
\end{aligned}
$$


for the stress function $\phi(r, z)$ occurring in Eqs. (11)-(15). Corresponding to this stress function we have the equations

$$
\begin{aligned}
& u=-\frac{(1-\nu) \epsilon}{1-2 \nu} \int_{0}^{\infty}\left[(1-2 \nu) \frac{\cosh \xi(\delta-\zeta)}{\sinh \xi \delta}-\xi \delta \frac{\cosh \xi \zeta}{\sinh ^{2} \xi \delta}\right. \\
& \left.-\xi \zeta \frac{\sinh \xi(\delta-\zeta)}{\sinh \xi \delta}\right] \psi(\xi) J_{1}(\xi \rho) d \xi, \\
& w=\frac{2(1-\nu)^{2} \epsilon}{1-2 \nu} \int_{0}^{\infty}\left[\sinh \xi(\delta-\zeta)+\xi \delta \frac{\sinh \frac{\xi \zeta}{\sinh } \xi \delta}{\xi}-\xi \zeta \cosh \xi(\delta-\zeta)\right] \frac{\psi(\xi) J_{0}(\xi \rho)}{\sinh \xi \delta} d \xi
\end{aligned}
$$

by which to determine the displacement field.

Further, if we substitute from Eq. (18) into Eq. (14) and put $\zeta=0$, we find that

$$
\left[\sigma_{z}\right]_{z=0}=-\frac{E(1-\nu) \epsilon}{(1+\nu)(1-2 \nu) d} \int_{0}^{\infty} \psi(\xi) K(\xi \delta) J_{0}(\xi \rho) d \xi
$$

where

$$
K(\xi \delta) \equiv \xi \delta[1+H(\xi \delta)]=\xi \delta \cdot \frac{\xi \delta+\sinh \xi \delta \cosh \xi \delta}{\sinh ^{2} \xi \delta} .
$$

If we substitute the conditions (3) and (5) into Eqs. (20) and (21) we find that the function $\psi(\xi)$ must satisfy the dual integral equations

$$
\begin{gathered}
\int_{0}^{\infty} \xi[1+H(\xi \delta)] \psi(\xi) J_{0}(\xi \rho) d \xi=\delta^{-1}, \quad 0 \leq \rho \leq 1, \\
\int_{0}^{\infty} \psi(\xi) J_{0}(\xi \rho) d \xi=0, \quad \rho>1 .
\end{gathered}
$$

The second of the two boundary value problems can be treated in a similar way. If we substitute from Eq. (16) into Eq. (14) and make use of the condition (10) we find that, in this case, the relation between $A(\xi)$ and $B(\xi)$ is now

$$
\left(\xi^{2} \delta^{2}-2 \nu \sinh ^{2} \xi \delta\right) A+(\xi \delta+\sinh \xi \delta \cosh \xi \delta) B=0
$$

which may be written in the form

$$
A(\xi)=\frac{(1-2 \nu)(1+\nu) F}{E} \chi(\xi), \quad B(\xi)=-\frac{(1-2 \nu)(1+\nu) F}{E} \cdot \frac{\xi^{2} \delta^{2}-2 \nu \sinh ^{2} \xi \delta}{\xi \delta+\sinh \xi \delta \cosh \xi \delta} \chi(\xi) .
$$

The dual integral equations to be satisfied by $\chi(\xi)$ are then

$$
\begin{aligned}
\int_{0}^{\infty} \xi M(\xi \delta) \chi(\xi) J_{0}(\xi \rho) d \xi=1, & 0 \leq \rho \leq 1, \\
\int_{0}^{\infty} \chi(\xi) J_{0}(\xi \rho) d \xi=0, & \rho \geq 1,
\end{aligned}
$$

where

$$
M(\xi \delta)=\frac{\sinh ^{2} \xi \delta-\xi^{2} \delta^{2}}{\xi \delta+\cosh \xi \delta \sinh \xi \delta} .
$$

5. Solution of the dual integral equations. In this section we shall derive the solution of the pair of dual integral equations (23), (24) using the method of Lebedev and Uflyand 
[8]. Equations (25), (26) can be solved by a similar procedure. The essence of the LebedevUflyand method is that it reduces the solution of a pair of dual integral equations to a Fredholm integral equation of the second kind which can then be solved by standard methods [10].

Since we are interested in values of $\delta$ much greater than 1 we write the function $H(\xi \delta)$ in the form

$$
H(\xi \delta)=2 \sum_{m=1}^{\infty}(1+2 m \xi \delta) e^{-2 m \xi \delta} .
$$

Following Lebedev and Uflyand we write

$$
\psi(\xi)=\int_{0}^{1} \phi(t) \sin (\xi t) d t, \quad \phi(0)=0 .
$$

It is easily shown that the equation (24) is satisfied identically by this representation of $\psi(\xi)$. Substituting from Eq. (29) into (23) we find that

$$
\begin{aligned}
& \int_{0}^{\infty} J_{0}(\xi \rho) d \xi \int_{0}^{1} \xi \phi(t) \sin (\xi t) d t+\int_{0}^{\infty} \xi H(\xi \delta) J_{0}(\xi \rho) d \xi \\
& \cdot \int_{0}^{1} \phi(t) \sin (\xi t) d t=\delta^{-1}, \quad 0 \leq \rho \leq 1 .
\end{aligned}
$$

Since $\phi(0)=0$ we see on integrating by parts that

$$
\int_{0}^{1} \xi \phi(t) \sin (\xi t) d t=-\phi(1) \cos \xi+\int_{0}^{1} \phi^{\prime}(t) \cos (\xi t) d t .
$$

Now

$$
\int_{0}^{\infty} J_{0}(\xi \rho) \cos (\xi t) d t= \begin{cases}0, & 0<\rho<t \\ \left(\rho^{2}-t^{2}\right)^{-1 / 2}, & \rho>t,\end{cases}
$$

so that, by a simple change in the orders of integration we find that the first term on the left side of Eq. (30) becomes

$$
\int_{0}^{\rho}\left(\rho^{2}-t^{2}\right)^{-1 / 2} \phi^{\prime}(t) d t .
$$

If in the second term on the left side of Eq. (30) we replace $J_{0}(\xi \rho)$ by its integral representation

$$
(2 / \pi) \int_{0}^{\rho}\left(\rho^{2}-u^{2}\right)^{-1 / 2} \cos (u \xi) d u,
$$

we find that this term reduces to

$$
\frac{2}{\pi} \int_{0}^{\rho} \frac{d u}{\sqrt{\left(\rho^{2}-u^{2}\right)}} \int_{0}^{1} \frac{1}{2}\left[H^{*}(u+t)-H^{*}(u-t)\right] \phi(t) d t,
$$

where

$$
H^{*}(w)=\delta \int_{0}^{\infty} \xi H(\xi \delta) \sin (\xi w) d \xi
$$


We thus obtain the equation

$$
\begin{aligned}
\int_{0}^{\rho}\left(\rho^{2}-t^{2}\right)^{-1 / 2} \phi^{\prime}(t) d t+(2 / \pi \delta) \int_{0}^{\rho} & \left(\rho^{2}-u^{2}\right)^{-1 / 2} d u \\
& \cdot \int_{0}^{1} \phi(t) K_{1}(u, t) d t=\delta^{-1}, \quad 0 \leq \rho \leq 1,
\end{aligned}
$$

where $K_{1}(u, t)=\frac{1}{2}\left[H^{*}(u+t)-H^{*}(u-t)\right]$. We now let

$$
\int_{0}^{\rho}\left(\rho^{2}-t^{2}\right)^{-1 / 2} \phi^{\prime}(t) d t=f(\rho) ;
$$

then it is well-known ([10], p. 39) that

$$
\phi^{\prime}(t)=\frac{2}{\pi} \frac{d}{d t} \int_{0}^{t} v\left(t^{2}-v^{2}\right)^{-1 / 2} f(v) d v
$$

and since $\phi(0)=0$ we may integrate to obtain the expression

$$
\phi(t)=\frac{2}{\pi} \int_{0}^{t} \frac{v f(v) d v}{\sqrt{\left(t^{2}-v^{2}\right)}} .
$$

If we substitute this expression into Eq. (33), change the order of the integrations and write

$$
K(\rho, v)=v \int_{0}^{1}\left(t^{2}-v^{2}\right)^{-1 / 2} d t \int_{0}^{\rho}\left(\rho^{2}-u^{2}\right)^{-1 / 2} \delta^{2} K_{1}(u, t) d u, \quad 0<\rho<1
$$

we find that Eq. (33) reduces to the Fredholm equation

$$
f(\rho)+4 \pi^{-2} \delta^{-3} \int_{0}^{1} f(v) K(\rho, v) d v=\delta^{-1}, \quad 0<\rho<1 .
$$

By the classical theory of these equations ([10], p. 49) we can obtain a solution of this equation as a power series in $\left(4 \pi^{-2} \delta^{-3}\right)$ provided that $\delta$ is sufficiently large for $\frac{1}{4} \pi^{2} \delta^{3}>M$, where $M$ is the bound on the kernel $K(\rho, \nu)$. In this way we obtain the solution

$f(\rho)=\delta^{-1}\left\{1-4 \pi^{-2} \delta^{-3} \int_{0}^{1} K(\rho, v) d v+\left(4 \pi^{-2} \delta^{-3}\right)^{2} \int_{0}^{1} \int_{0}^{1} K(\rho, v) K(v, w) d v d w+\cdots\right\}$.

Since $K(\rho, v)$ is of order 0 in $\delta$ this means that

$$
f(\rho)=\delta^{-1}\left\{1-4 \pi^{-2} \delta^{-3} \int_{0}^{1} K(\rho, v) d v\right\}+0\left(\delta^{-7}\right) .
$$

To obtain the solution of Eq. (37) to this order we need only calculate

$$
\int_{0}^{1} K(\rho, v) d v=\int_{0}^{\rho}\left(\rho^{2}-w^{2}\right)^{-1 / 2} d w \int_{0}^{1} t\left[\delta^{2} K_{1}(w, t)\right] d t .
$$

Using the expansion (28) for $H$ we find from Eq. (32) that

$$
H^{*}(u)=\frac{1}{2} \delta^{-2} \sum_{m=1}^{\infty} m^{-3}\left\{u\left[1+(u / 2 m \delta)^{2}\right]^{-2}+u\left[1+(u / 2 m \delta)^{2}\right]^{-3}\left[3-(u / 2 m \delta)^{2}\right]\right\}
$$


so that

$$
\kappa(w) \equiv \int_{0}^{1} t\left[\delta^{2} K_{1}(w, t)\right] d t=\sum_{m=1}^{\infty} m^{-3}\left(I_{1}-I_{2}+I_{3}-I_{4}\right),
$$

where

$$
\begin{gathered}
I_{1}=\frac{1}{4} \int_{0}^{1} \frac{t(w+t) d t}{\left[1+\{(w+t) / 2 m \delta\}^{2}\right]^{2}}=m^{3} \delta^{3}\left[\tan ^{-1}\{(w+1) / 2 m \delta\}\right. \\
\left.-\tan ^{-1}(w / 2 m \delta)\right]-\frac{1}{2} m^{2} \delta^{2}\left[1+\{(w+1) / 2 m \delta\}^{2}\right]^{-1}, \\
\begin{array}{r}
I_{2}=\frac{1}{4} \int_{0}^{1} \frac{t(w-t) d t}{\left[1+\{(w-t) / 2 m \delta\}^{2}\right]^{2}}=m^{3} \delta^{3}\left[\tan ^{-1}\{(w-1) / 2 m \delta\}\right. \\
\left.-\tan ^{-1}(w / 2 m \delta)\right]+\frac{1}{2} m^{2} \delta^{2}\left[1+\{(w-1) / 2 m \delta\}^{2}\right]^{-1}, \\
I_{3}=\frac{1}{4} \int_{0}^{1} \frac{t(w+t)\left[3-\{(w+t) / 2 m \delta\}^{2}\right] d t}{\left[1+\{(w+t) / 2 m \delta\}^{2}\right]^{3}}=\frac{1}{8}\left[\left\{(w+2)(w+1)^{2}+4 w m^{2} \delta^{2}\right\}\right. \\
\left.\cdot\left\{1+[(w+1) / 2 m \delta]^{2}\right\}^{-2}-\left(w^{3}+4 w m^{2} \delta^{2}\right)\left[1+w^{2} / 4 \delta^{2} m^{2}\right)^{-1}\right], \\
I_{4}=\frac{1}{4} \int_{0}^{1} \frac{t(w-t)\left[3-\{(w-t) / 2 m \delta\}^{2}\right] d t}{\left[1+\{(w-t) / 2 m \delta\}^{2}\right]^{3}}=\frac{1}{8}\left[\left\{(w-2)(w-1)^{2}+4 w m^{2} \delta^{2}\right\}\right. \\
\left.\cdot\left\{1+[(w-1) / 2 m \delta]^{2}\right\}^{-2}-\left(w^{3}+4 w m^{2} \delta^{2}\right)\left(1+w^{2} / 4 \delta^{2} m^{2}\right)^{-1}\right] .
\end{array}
\end{gathered}
$$

Since the solution (38) is valid only to order $\delta^{-6}$ we replace $I_{1}, I_{2}, I_{3}, I_{4}$ by their expansions in powers of $\delta$ up to $\delta^{-2}$. We find that

$$
\kappa(w)=\frac{2}{3} \zeta(3)-\frac{3}{20} \delta^{-2} \zeta(5)\left(5 w^{2}+2\right)+0\left(\delta^{-4}\right),
$$

where

$$
\zeta(3)=\sum_{m=1}^{\infty} m^{-3}=1.202, \quad \zeta(5) \equiv \sum_{m=1}^{\infty} m^{-5}=1.0369 .
$$

The numerical values of the zeta-functions $\zeta(3), \zeta(5)$ are taken from [11]. Substituting from Eqs. (40) and (41) into Eq. (38) we obtain the expression

$$
f(\rho)=\delta^{-1}\left[1-4 \zeta(3) /\left(3 \pi \delta^{3}\right)+3 \zeta(5)\left(5 \rho^{2}+4\right) /\left(20 \pi \delta^{5}\right)+0\left(\delta^{-7}\right)\right] .
$$

From Eqs. (35), (29) we find that

$$
\begin{aligned}
\psi(\xi) & =(2 / \pi) \int_{0}^{1} \sin \xi t d t \int_{0}^{t} \rho\left(t^{2}-\rho^{2}\right)^{-1 / 2} f(\rho) d \rho \\
& =-(2 / \pi)\left[f_{0} D-\frac{2}{3} f_{1} D^{3}\right] \xi^{-1} \sin \xi+0\left(\delta^{-7}\right),
\end{aligned}
$$

where

$$
f_{0}=\delta^{-1}\left\{1-4 \zeta(3) /\left(3 \pi \delta^{3}\right)+3 \zeta(5) /\left(5 \pi \delta^{5}\right)\right\}, \quad f_{1}=3 \zeta(5) /\left(4 \pi \delta^{5}\right)
$$

and $D$ denotes the operator $d / d \xi$.

6. Shape of the deformed crack. We shall now proceed to find an approximate expression-correct to order $\delta^{-6}$-for the displacement $w$ on $z=0,0 \leq \rho \leq 1$. Putting 
$\zeta=0$ in Eq. (20) we find that the normal component of the surface displacement is given by the equation

$$
w_{0}(\rho)=\frac{2(1-\nu)^{2}}{1-2 \nu} \epsilon \int_{0}^{\infty} \psi(\xi) J_{0}(\xi \rho) d \xi
$$

which by Eq. (29) and the result

$$
\int_{0}^{\infty} J_{0}(\xi \rho) \sin (\xi t) d \xi= \begin{cases}0, & t<\rho \\ \left(t^{2}-\rho^{2}\right)^{-1 / 2}, & t>\rho\end{cases}
$$

we find that

$$
w_{0}(\rho)=2(1-\nu)^{2}(1-2 \nu)^{-1} \epsilon \int_{\rho}^{1}\left(t^{2}-\rho^{2}\right)^{1 / 2} \phi(t) d t
$$

which, because of (35) can be written in the form

$$
w_{0}(\rho)=4(1-\nu)^{2} \epsilon /[\pi(1-2 v)] \int_{\rho}^{1}\left(t^{2}-\rho^{2}\right)^{-1 / 2} d t \int_{0}^{t} v\left(t^{2}-v^{2}\right)^{-1 / 2} f(v) d v .
$$

Substituting for $f(v)$ from Eq. (42) and performing the integrations, we find that

$$
\begin{aligned}
w_{0}(\rho)=\frac{4(1-\nu)^{2}}{\pi(1-2 \nu)}\left(\frac{\epsilon}{\delta}\right)\left(1-\rho^{2}\right)^{1 / 2} & \\
\cdot & \left\{1-[4 \zeta(3)] /\left(3 \pi \delta^{3}\right)-\left[\zeta(5)\left(23+10 \rho^{2}\right)\right] /\left(30 \pi \delta^{5}\right)+0\left(\delta^{-7}\right)\right\} .
\end{aligned}
$$

Using the numerical values of the zeta-functions we find that

$$
w_{0}(\rho)=\left\{4(1-\nu)^{2} \epsilon /[\pi \delta(1-2 \nu)]\right\} \cdot\left(1-\rho^{2}\right)^{1 / 2} k(\rho, \delta),
$$

where "the correction term" $k(\rho, \delta)$ is defined by the equation

$$
k(\rho, \delta)=1-0.510 \delta^{-3}-0.011 \delta^{-5}\left(23+10 \rho^{2}\right)+0\left(\delta^{-7}\right) .
$$

\section{REFERENCES}

1. G. R. Irwin, Fracture, Handbuch der Physik 6, 1958, pp. 551-590

2. G. I. Barenblatt, The Formation of equilibrium cracks during brittle fracture, J. Appl. Math. \& Mechanics 23, 622 (1959) [English translation of Prik. Mat. i Mech., 23, 434 (1959)]

3. I. N. Sneddon, The distribution of stress in the neighbourhood of a crack in an elastic solid, Proc. Roy. Soc. A. 187, 229 (1946)

4. A. E. Green, On Boussinesq's problem and penny-shaped cracks, Proc. Cambridge Phil. Soc. 45. 251 (1948)

5. I. N. Sneddon, Elements of partial differential equations, McGraw-Hill, New York, 1957

6. L. E. Payne, On axially symmetric punch, crack and torsion problems, J. Soc. Ind. Appl. Math. 1, $53(1953)$

7. R. A. Sack, Extension of Griffith's theory of rupture to three dimensions, Proc. Phys. Soc. 58, 729 (1946)

8. N. N. Lebedev and Ya. S. Uflyand, Axisymmetric contact problem for an elastic layer, J. Appl. Math. \& Mech. 22, 442 (1958) [English translation of Prik. Mat. i Mech., 22, 320 (1958)]

9. I. N. Sneddon, Fourier transforms, McGraw-Hill, New York, 1951

10. F. G. Tricomi, Integral equations, Interscience, New York, 1957

11. E. Jahnke and F. Emde, Tables of functions with formulae and curves, Dover, New York, 1943 